\begin{tabular}{|c|c|}
\hline Title & Tough Double-Network Gels and Elastomers from the Nonprestretched First Network \\
\hline Author(s) & $\begin{array}{l}\text { Nakajima, Tasuku; Ozaki, Y uhei; Namba, Ryo; Ota, Kumi; Maida, Y uki; Matsuda, Takahiro; Kurokawa, Takayuki; } \\
\text { Gong, Jian Ping }\end{array}$ \\
\hline Citation & $\begin{array}{l}\text { A CS Macro Letters, 8(11), 1407-1412 } \\
\text { https://doi.org/10.1021/acsmacrolett.9b00679 }\end{array}$ \\
\hline Issue Date & 2019-11-19 \\
\hline Doc URL & http:/hdl.handle.net/2115/79770 \\
\hline Rights & $\begin{array}{l}\text { This document is the A ccepted Manuscript version of a Published Work that appeared in final form in A CS Macro } \\
\text { Letters, copyright c A merican Chemical Society after peer review and technical editing by the publisher. To access the } \\
\text { final edited and published work see https://pubs.acs.org/doi/10.1021/acsmacrolett.9b00679. }\end{array}$ \\
\hline Type & article (author version) \\
\hline File Information & ACS Macro Letters_8(11).pdf \\
\hline
\end{tabular}

Instructions for use 


\title{
Tough Double-Network Gels and Elastomers from the Non-Pre- stretched First Network
}

\author{
Tasuku Nakajima*, a,b,c Yuhei Ozaki, ${ }^{\mathrm{d}}$ Ryo Namba, ${ }^{\mathrm{d}}$ Kumi Ota ${ }^{\mathrm{d}}$ Yuki Maida, ${ }^{\mathrm{d}}$ Takahiro Matsuda, ${ }^{\mathrm{a}}$ Taka- \\ yuki Kurokawa, a,b and Jian Ping Gong a,b,c \\ ${ }^{a}$ Faculty of Advanced Life Science, Hokkaido University, N21W11, Kita-ku, Sapporo, Hokkaido, 001-0021, Japan. \\ ${ }^{\mathrm{b} S o f t ~ M a t t e r ~ G I-C o R E, ~ H o k k a i d o ~ U n i v e r s i t y, ~ N 21 W 11, ~ K i t a-k u, ~ S a p p o r o, ~ H o k k a i d o, ~ 001-0021, ~ J a p a n . ~}$ \\ ${ }^{\mathrm{c}}$ WPI-ICReDD, Hokkaido University, N21W10, Kita-ku, Sapporo, Hokkaido, 001-0021, Japan. \\ ${ }^{\mathrm{d}}$ Graduate School of Life Science, Hokkaido University, N10W8, Kita-ku, Sapporo, Hokkaido, 060-0810, Japan.
}

\begin{abstract}
Double-network (DN) gels and elastomers, which consist of two (or more) rubbery polymer networks with contrasting physical properties, have received significant attention as they are extremely tough soft materials. The $1^{\text {st }}$ network of tough DN materials should be more brittle and weaker than the $2^{\text {nd }}$ network. In this paper, we reexamined the structural requirements of the covalently-cross-linked $1^{\text {st }}$ network of tough DN materials and established a non-prestretching strategy. While prestretching of network strands has been considered necessary for preparation of the brittle and weak $1^{\text {st }}$ network, we found that a non-prestretched network having a short strand length and low strand density can be used as the brittle and weak $1^{\text {st }}$ network for preparation of both tough DN gels and elastomers. This work can further expand the chemical and mechanical diversity of DN materials.
\end{abstract}

Elastomers and gels, which are rubbery polymeric materials, have been widely used as industrial or medical materials owing to their unique softness. Examples include tires and stretchable electronics (elastomers) [1], contact lenses and cell cultivation scaffolds (gels) [2-3], and so on. Recently, various toughening methods for the rubbery materials have been introduced to improve their durability [4-5]. Specifically, the double network (DN) strategy has attracted much attention as an effective toughening method for both gels [6-11] and elastomers [11-15]. For example, the optimized DN gels show extremely high fracture energy of $4,000 \mathrm{~J} \mathrm{~m}^{-2}$, whereas conventional gels show fracture energy of 1-100 $\mathrm{J} \mathrm{m}^{-2}$ [10].

Tough DN gels or elastomers are interpenetrating network materials, consisting of the contrasting two (or more) networks. Combinations of the two networks of DN materials that give high toughness are strongly limited $[6,11]$. The following two conditions are considered important for the effective toughening:

1. The $1^{\text {st }}$ network is more "brittle (low extensibility)" than the $2^{\text {nd }}$ network [7]. Deformation ratio of a network at break, $\lambda_{\mathrm{f}}$, can be used as an index of its brittleness.

2. The $1^{\text {st }}$ network is "weaker" than the $2^{\text {nd }}$ network [9]. Tensile nominal fracture stress, $\sigma_{\mathrm{f}}$, can be an index of its weakness.

When a tough DN material having such contrasting two networks is deformed, as shown in Figure 1(a), the brittle strands of the $1^{\text {st }}$ network firstly rupture, while the stretchable $2^{\text {nd }}$ network keeps unity of the material. During the deformation process, massive $1^{\text {st }}$ network strands get ruptured and dissipate large energy before fracture of the stretchable $2^{\text {nd }}$ network, which leads extremely high toughness of a DN material [16-18]. Such $1^{\text {st }}$ network fracture in wide range visually appears as yielding-like phenomenon and irreversible mechanical hystere- sis during tensile test [7, 16-18]. Here we emphasize again importance of the above two conditions. If condition 1 is not satisfied, the two networks having similar extensibility fracture almost simultaneously like a single network gel. If condition 2 is not satisfied, after rupture of the $1^{\text {st }}$ network strands, the $2^{\text {nd }}$ network strands cannot sustain the transferred force, leading immediate macroscopic fracture of both the networks.

However, what are the structural requirements of the $1^{\text {st }}$ network which satisfies these two conditions (small $\lambda_{\mathrm{f}}$ and $\sigma_{\mathrm{f}}$ )? Although the tough DN gels can be made from both chemicallyand physically-cross-linked brittle $1^{\text {st }}$ network [19-21], in this work we focus on the DN gels with the chemically-cross-linked $1^{\text {st }}$ network. Let us assume a homogeneous chemically-crosslinked network with the Kuhn segment concentration, $c$, and the network strand density, $n$, as the $1^{\text {st }}$ network of a DN material. Average number of Kuhn segments per a network strand, $N$, of this network is $c / n$ without consideration of trapped entanglement. The average end-to-end distance of its network strands is expressed as $b N^{v}$, where $b$ is Kuhn length and $v$ is Flory exponent, which relates to the degree of expansion of the strands. For condition $1, \lambda_{\mathrm{f}}$ of a gel is close to maximum deformation ratio of its network strands [22, 23]. As shown in Figure 1(b), maximum deformation ratio of a single strand is the ratio of its contour length, $b N^{1}$, to the end-to-end distance at its unperturbed state, $b N^{v}$. Thus, $\lambda_{\mathrm{f}}$ of a gel is approximately described as

$$
\lambda_{f} \approx \frac{b N^{1}}{b N^{v}}=N^{1-v}=\left(\frac{c}{n}\right)^{1-v}
$$

For condition 2, $\sigma_{\mathrm{f}}$ should be approximately proportional to the area density of the $1^{\text {st }}$ network strands at the relaxed state [8, 24]. In particular,

$$
\sigma_{f} \propto n^{\frac{2}{3}}
$$


(a) Tough double network (DN) materials

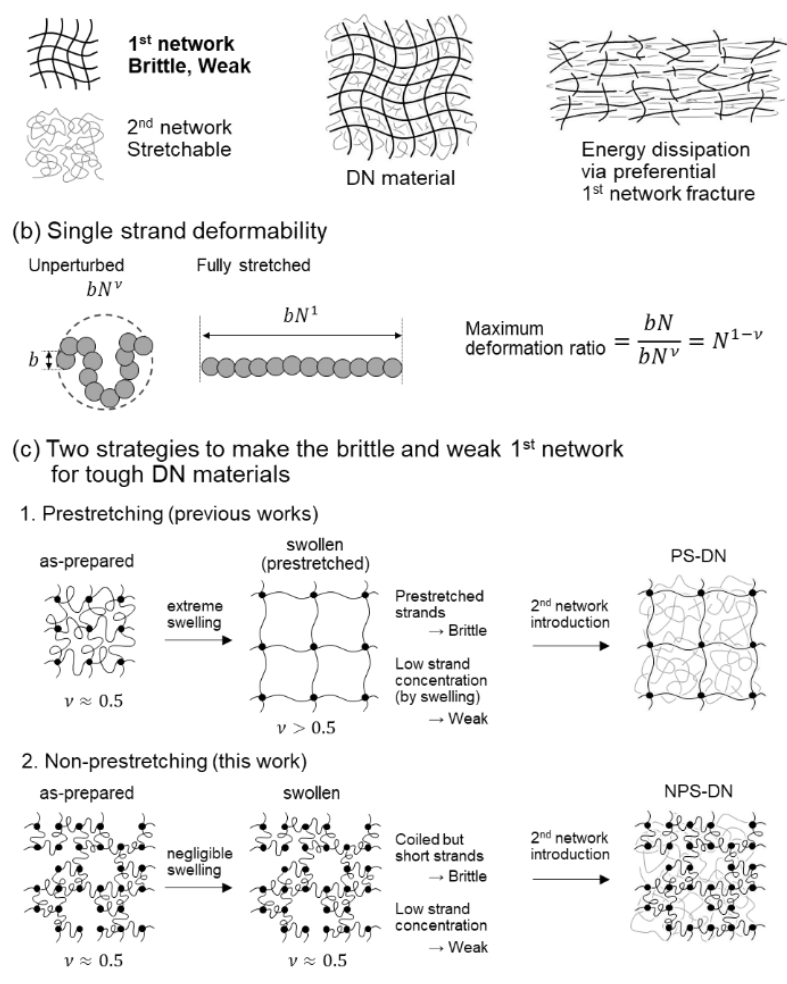

Figure 1. (a) Double-network materials consisting of the brittle and weak $1^{\text {st }}$ network and stretchable $2^{\text {nd }}$ network, and their energy dissipation mechanism. (b) Theoretical maximum deformation ratio of single network strand with Kuhn length $b$, Kuhn segment number $N$, and Flory exponent $v$. (c) The two strategies to make the brittle and weak $1^{\text {st }}$ network for tough DN materials.

Eq. (1) suggests that either large $v$ or small $N(=c / n)$ is necessary to make the network "brittle". Eq. (2) implies lower strand density $n$ is necessary to make the network "weak". Therefore, there should be two possible strategies to make the brittle and weak $1^{\text {st }}$ network, as shown in Figure 1(c). The first one, so-called prestretching strategy, is to use the highly swollen, prestretched $1^{\text {st }}$ network to achieve large $v$ and small $n$. Most of the reported DN materials with the chemically-crosslinked $1^{\text {st }}$ network have adopted this strategy. A polyelectrolyte (PE) network is typically used as the prestretchable $1^{\text {st }}$ network $[6,14]$. A PE gel swells largely in polar solvents as dissociated counter-ions of the PE gel possess large osmotic pressure of the gel. As a result, a swollen PE gel has small $c$ and $n$ because the swelling induces dilution of the network. In addition, a swollen PE gel has large $v$ due to swelling-induced prestretching of their strands. Large $v$ leads small $\lambda_{f}$, which satisfies the condition 1 , whereas small $n$ leads small $\sigma_{\mathrm{f}}$, which satisfies the condition 2 . Thus, a PE network can be the $1^{\text {st }}$ network of tough DN materials. This prestretching strategy has been also applied to the neutral polymer (NP)-based $1^{\text {st }}$ network. Since an NP gel does not swell much in solvents due to lack of counter-ions, it exhibits a small $v$ of $0.5-0.6$ even at its swollen state. To increase the swelling ratio of an NP gel, additional components can be introduced to the NP network to increase its entire osmotic pressure. For example, introduction of linear polyelectrolytes or ionic micelles (molecular stent method) effectively increases overall osmotic pressure and swelling ratio of a NP gel [8]. Such highly swollen NP gels having large $v$ and small $n$ can be used as the $1^{\text {st }}$ network of a tough DN materials. Additionally, triple network method has been proposed based on the similar idea [12-13,25-26]. In this method, the neutral but concentrated $2^{\text {nd }}$ network possesses large osmotic pressure to promote the $1^{\text {st }}$ network swelling, and the stretchable $3^{\text {rd }}$ network acts as the ductile network.

The other possible strategy to fabricate the brittle and weak $1^{\text {st }}$ network is to synthesize networks having small segment number per a strand $N(=c / n)$ and small network strand density $n$ without prestretching of the strands. $N$ can be decreased by increasing $n$ or decreasing $c$. Increase of $n$, however, causes increase of $\sigma_{\mathrm{f}}$, which is incompatible with condition 2 . Therefore, $N$ should be decreased mainly by decreasing Kuhn segment concentration $c$ while keeping strand density $n$ low. The small $N=c / n$ (short strand length) makes the gels extremely brittle, which satisfies the condition 1 . Its subdued strand density $n$ makes the gels sufficiently weak, which satisfies the condition 2.

Although these two strategies can be theoretically used for fabrication of tough DN materials with the chemicallycrosslinked $1^{\text {st }}$ network, to the best of our knowledge, no study has adopted the latter strategy. Here, the purpose of this study is to experimentally confirm validity of the non-prestretching strategy for synthesis of the brittle and the weak $1^{\text {st }}$ network for both tough DN gels and elastomers. First, we tried synthesis of tough DN gels with the non-prestretched $1^{\text {st }}$ network synthesized by random copolymerization of monomer and cross-linker. To achieve low Kuhn segment concentration $c$ of the network, we used low monomer concentration for the gel preparation. Also, to achieve short strand length $c / n$, molar ratio of the cross-linker to the monomer was kept sufficiently high. In particular, the $1^{\text {st }}$ network was synthesized from the aqueous solutions containing $0.2-1 \mathrm{M}$ of $N, N$-dimethylacrylamide (DMAAm) as a monomer and 3-21 mol\% (to monomer) of $N, N$ '-methylenebis(acrylamide) (MBAA) as a cross-linker. Note that the gelation reaction should not be ideal at such extreme low monomer concentration and high cross-linking ratio. The obtained networks may contain many defects, and some cross-linkers may not work as cross-linking points (connecting 3 or more strands) but substantially work as monomers (connecting 2 strands). In fact, the lowest monomer concentration of $0.2 \mathrm{M}$ is near the critical concentration for gelation, below which bulk gels could not be synthesized. Subsequently, the ductile $2^{\text {nd }}$ polyacrylamide (PAAm) network was synthesized in the presence of the $1^{\text {st }}$ network. The obtained PDMAAm/PAAm DN gels with the non-prestretched $1^{\text {st }}$ network are denoted as NPS-DN $(x-y)$ gels, where $x$ and $y$ are feed monomer concentration (M) corresponding to $c$ and ratio of the cross-linker to the monomer (mol\%) corresponding to $c / n$, respectively. Thickness ratio of the $1^{\text {st }}$ PDMAAm network in the NPS-DN gels to its as-prepared state, $\alpha$, was around 1.1, suggesting the PDMAAm network is not prestreched in the NPS-DN gels (Supporting Information). As a control, the conventional DN gels with the prestretched poly(2-acrylamido-2methylpropanesulfonic acid) (PAMPS), $1^{\text {st }}$ network (PS-DN gels) were also prepared. $\alpha$ of the PAMPS network was 2.3, suggesting the network is highly prestretched. The obtained NPS-DN and PS-DN gels were tested without soaking in water unless specially mentioned. 


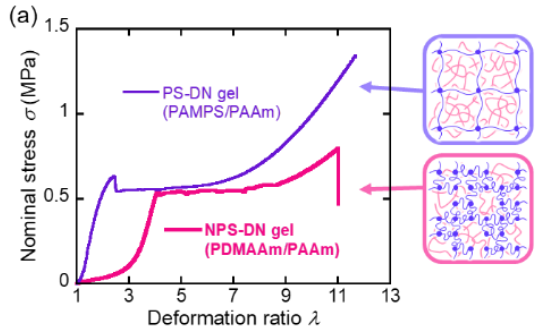

(b)
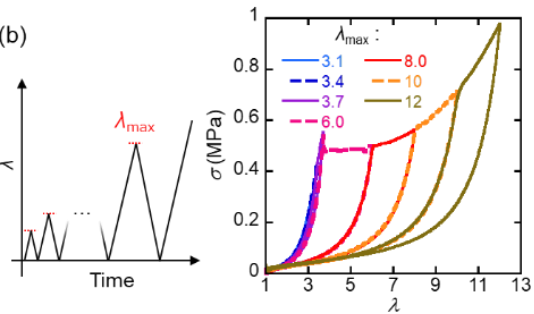

(c)

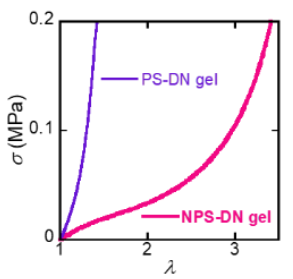

Figure 2. (a) Nominal tensile stress-deformation ratio $(\sigma-\lambda)$ curves of the typical prestretched (PS)-DN gel and the non-prestretched (NPS)-DN(0.4-8) gel. (b) Cycle tensile test results of the NPS$\mathrm{DN}(0.4-8)$ gel with the deformation ratio-time diagram. (c) Initial $\sigma-\lambda$ curves of the PS-DN and NPS-DN gels.

Figure 2(a) shows nominal tensile stress-deformation ratio $(\sigma-\lambda)$ curves of the typical NPS-DN and the PS-DN gels. The PS-DN gel showed high strength and stretchability as well as clear yielding. The NPS-DN(0.4-8) gel also displayed high mechanical performance like the PS-DN gel, such as high fracture stress of $0.8 \mathrm{MPa}$ and high deformation ratio at break of 11 with clear yielding. Moreover, the NPS-DN gels possess high mechanical performance even after swollen in water (Supporting Information). As shown in Figure 2(b), the NPS-DN gel presented irreversible energy dissipation upon cycle tensile test, like PS-DN gels [16-18]. It suggests that the non-prestretched $1^{\text {st }}$ network in the NPS-DN gels works as brittle sacrificial bonds to dissipate large energy during deformation, which contributes to high toughness of the DN gels. These results show validity of the non-prestretching strategy for preparation of tough DN gels. On the other hand, at small deformation, the mechanical response of the PS-DN and NPS-DN gels are quite different. As shown in Figure 2(c), the NPS-DN gel is initially soft and shows strain hardening at $\lambda>3$, whereas the conventional PS-DN gel is stiff from the beginning of the stretch. Regarding this, the initial stiffness of the NPS-DN gels is much smaller than the PS-DN gel despite higher cross-linking ratio of the NPS $1^{\text {st }}$ network. Moreover, the NPS-DN gel exhibits an Sshaped curve (softening-hardening) while the PS-DN gel shows a J-shaped curve (only hardening) at their low $\lambda$ regime. $\sigma-\lambda$ curves of DN materials, before yielding, are dominated by the $1^{\text {st }}$ network [13,27-28]. Typical rubbery materials having coiled strands show S-shaped curves [29]. As the non-prestretched $1^{\text {st }}$ network strands are in coiled state, an NPS-DN gel also exhibits a standard S-shaped curve with initial soft regime. However, the $1^{\text {st }}$ network of a PS-DN gel is highly pre-stretched. Thus, initial softening regime in a $\mathrm{S}$-shaped curve does not appear in the $\sigma-\lambda$ curve of the PS-DN gel, resulting in the Jshaped curve. Furthermore, the prestretched strands have higher elastic energy than the non-prestretched strands, resulting higher initial stiffness of the PS-DN gel than the NPS-DN gel. (a) NPS-DN $(x-8)$

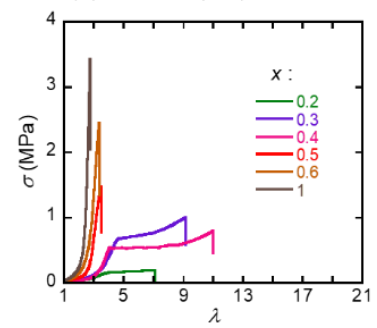

(b) NPS-DN $(0.3-y)$
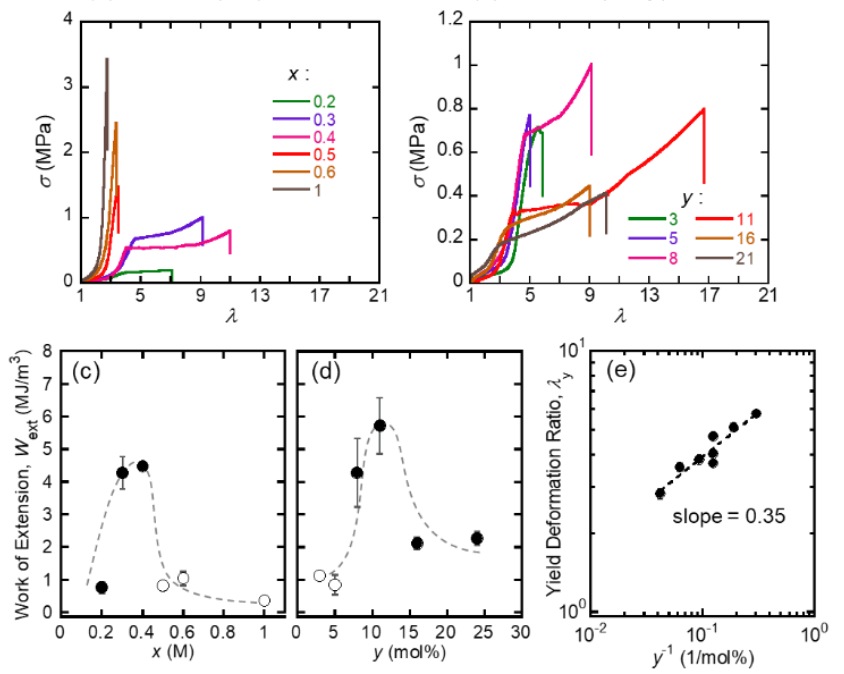

Figure 3. (a) $\sigma-\lambda$ curves of the NPS-DN $(x-8)$ gels. (b) $\sigma-\lambda$ curves of the NPS-DN (0.3-y) gels. (c) Work of extension of the NPS$\mathrm{DN}(x-8)$ and $(\mathrm{d})$ work of extension of the NPS-DN $(0.3-y)$ gels. The filled symbols denote the samples showing distinct yielding whereas the open symbols denote the samples without yielding. The dashed lines are guide for eyes. (e) Relationship between the yield deformation ratio and $y^{-1}$ of the NPS-DN $(x-y)$ gels. The small error bars are hidden by the symbols.

Effect of the $1^{\text {st }}$ network composition to mechanical properties of NPS-DN gels was evaluated. Initially, feed monomer concentration $x$ was varied but the ratio of cross-linker $y$ was fixed to $8 \mathrm{~mol} \%$. As $x$ and $y$ correspond to $c$ and $c / n$, respectively, varying $x$ while keeping $y$ same ideally corresponds to change of segment density $c$ and strand concentration $n$, with constant strand length $c / n$. Figure 3(a) depicts tensile $\sigma$ - $\lambda$ curves of the NPS-DN $(x-8)$ gels. When $x$ is too large $(x>0.4 \mathrm{M})$, the NPS-DN gels are strong but brittle due to too large $n$ (condition 2 is not satisfied). When $x \leq 0.4 \mathrm{M}$, the obtained NPS-DN gels show yielding due to appropriate $n$. Secondly, $x$ is fixed to 0.3 $\mathrm{M}$ and $y$ is varied. It ideally corresponds to vary $c / n$ and $n$ while keeping $c$ constant. Figure 3(b) shows tensile curves of NPSDN $(0.3-y)$ gels. When $y$ is too small, the NPS-DN gels are brittle due to too large strand length $c / n$ (condition 1 is not satisfied). The gels showed yielding and high toughness when $y \geq 6 \mathrm{~mol} \%$. Note that, the NPS-DN gel with $y \geq 16 \mathrm{~mol} \%$ was turbid and showed unclear yielding because of inhomogeneous network structure induced by large cross-linking ratio. When the toughness of the DN gels are simply evaluated by the area under $\sigma-\lambda$ curves (work of extension), it is clear that the NPS-DN gels which show yielding have significantly higher toughness than those without yielding, except the NPS-DN(0.2-8) gel (Figure $3(\mathrm{c}, \mathrm{d}))$.

It would be interesting to explore the relationship between the mechanical properties of the NPS-DN gels and their $1^{\text {st }}$ network structure. Our previous study implies that the yield deformation ratio $\lambda_{\mathrm{y}}$ of $\mathrm{DN}$ gels corresponds to the maximum stretching ratio of the $1^{\text {st }}$ network strands, $(c / n)^{1-v}$, as,

$$
\lambda_{y} \approx\left(\frac{c}{n}\right)^{1-v}
$$

Considering $y$ is ideally inversely proportional to $c / n$, we plotted $\lambda_{\mathrm{y}}$ of the as-prepared NPS-DN $(x-y)$ gels against $y^{-1}$ as shown in Figure 3(e). A positive power-law relationship with the slope of 
0.35 is found to exist between them. The slope $(\approx 1-v)$ of 0.35 corresponds to $v$ of 0.65 , which is close to theoretical $v$ of $0.5-$ 0.6 .

Subsequently, we applied this non-prestretching strategy to preparation of tough NPS-DN elastomers. Following the previous reports on DN elastomers, we adopt poly(ethyl acrylate) (PEA) network as the stretchable $2^{\text {nd }}$ network [12-15]. Apart from the two requirements for the $1^{\text {st }}$ network of DN materials, there are three additional requirements for the brittle, non-prestretched $1^{\text {st }}$ network for DN elastomers: 1$)$ Tg of the $1^{\text {st }}$ network is lower than $0{ }^{\circ} \mathrm{C}, 2$ ) bulk ethyl acrylate is a good solvent for the $1^{\text {st }}$ network, 3 ) the monomeric units should be dissolvable in water (because water is the desired solvent for the network synthesis at such extremely-low monomer concentration owing to its negligible chain transfer reaction constant [30]). Polyethylene glycol diacrylate (PEGDA) was chosen as the monomeric units for the $1^{\text {st }}$ network which conform to these additional requirements. We synthesized the poly(PEGDA) (PPEGDA) hydrogels from the PEGDA aqueous solutions with weight concentration of 10-30 wt\%. Tg of the dried PPEGDA network was determined by the differential scanning calorimeter as $-40^{\circ} \mathrm{C}$ (data not shown). The PPEGDA hydrogels were dehydrated and immersed in ethyl acrylate liquid containing cross-linker. Then, the PEA network was synthesized in the presence of the PPEGDA networks. The obtained PPEGDA/PEA NPS-DN elastomers are denoted as NPS-DN $(z)$ elastomers, where $z$ is feed PEGDA concentration (wt $\%$ ).
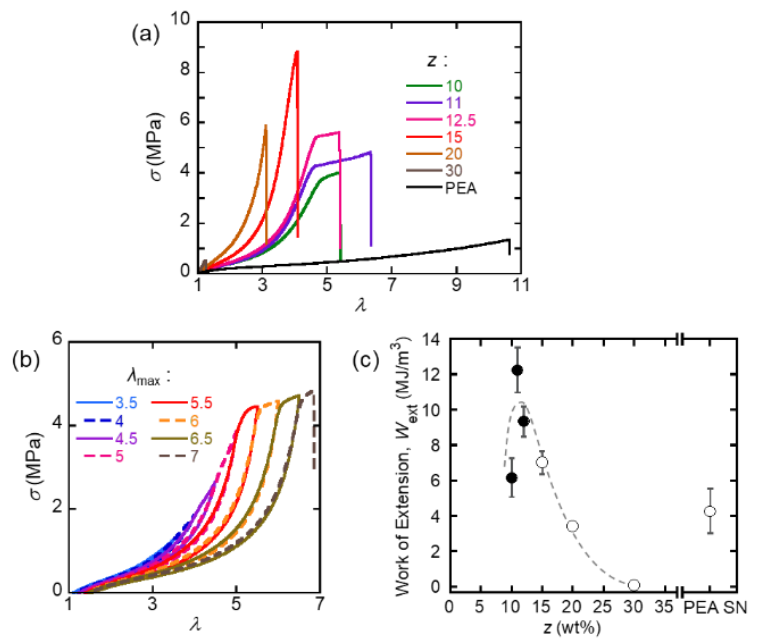

Figure 4. (a) $\sigma-\lambda$ curves of the PPEGDA/PEA NPS-DN(z) elastomers and the PEA single network elastomer. (b) Cycle tensile test results of the NPS-DN(11) elastomer. (c) Work of extension of the NPS-DN $(z)$ elastomers and the PEA elastomer. The filled and open symbols denote the samples with and without yielding, respectively. The dashed line is a guide for eyes.

Figure 4(a) shows effect of the PEGDA feed concentration $z$ on the $\sigma-\lambda$ curves of the PPEGDA/PEA NPS-DN $(z)$ elastomers. . When $z \geq 15 \mathrm{wt} \%$, the NPS-DN $(z)$ elastomers were brittle without yielding. On the other hand, the NPS-DN elastomers show yielding when $z \leq 12 \mathrm{wt} \%$. At this condition, the NPS-DN elastomers show irreversible energy dissipation upon their cycle tensile test like DN gels (Figure 4(b)). Toughness (work of extension) of the NPS-DN elastomers with yielding was improved significantly in comparison with that of the PEA single network elastomer, as shown in Figure 4(c). These results clarify that the non-prestretching method can be principally applied to fabricate tough DN elastomers. Optimization of preparation conditions for stronger and tougher DN elastomers is a future challenge.

In conclusion, we have established the novel non-prestretching strategy for preparation of tough DN gels and elastomers in addition to the conventional prestretching strategy, based on the theoretical investigation of structural requirements for tough DN materials. As PS-DN and NPS-DN materials commonly have high toughness but show different initial mechanical response, we can choose the appropriate one in accordance to the specific application. For example, NPS-DN materials with initial soft regime should be more suitable for sealing materials and stretchable electronics than rigid PS-DN materials. Moreover, this study can expand chemical diversity of DN materials. While conventional prestretching strategy basically requires the polyelectrolyte $1^{\text {st }}$ network, this non-prestretching methodology enables to use more general neutral network as the $1^{\text {st }}$ network. Since use of polyelectrolytes is harmful to biological tissues in some cases [31], the polyelectrolyte-free tough DN gels, realized by this study, can be desired medical materials.

\section{Experimental Section.}

For preparation of the non-prestretched $1^{\text {st }}$ network, $x \mathrm{M}$ of DMAAm, $y$ mol\% of MBAA (to monomer), and $5 \mathrm{mM}$ of potassium persulfate were dissolved in pure water. $5 \mathrm{mM}$ of $N, N, N^{\prime}, N^{\prime}$-tetramethylethylelediamine was then added to the solutions to initiate gelation. The solutions were immediately poured into the glass molds prepared with the two soda-lime glass plates (thickness: $3 \mathrm{~mm}$ ) and the silicone rubber (thickness: $1 \mathrm{~mm}$ ) as a spacer and kept for more than $24 \mathrm{~h}$ at $4{ }^{\circ} \mathrm{C}$ in air to synthesize the PDMAAm gels. For preparation of the prestretched network, $1 \mathrm{M}$ of AMPS, $40 \mathrm{mM}$ of MBAA, and 10 $\mathrm{mM}$ of 2-oxogultaric acid were dissolved in pure water. The solution was then poured into the glass mold. $365 \mathrm{~nm}$ UV (4 $\mathrm{mW} / \mathrm{cm}^{2}$ ) polymerization was carried out for $7 \mathrm{~h}$ to synthesize the PAMPS gel in an argon blanket. The 1st network gels were immersed in the excess $2^{\text {nd }}$ network precursor aqueous solutions containing $4 \mathrm{M}$ of acrylamide (AAm), $0.4 \mathrm{mM}$ of MBAA, and $0.4 \mathrm{mM}$ of 2-oxogultaric acid for more than $24 \mathrm{~h}$ and then sandwiched with the two glass plates. After that, $365 \mathrm{~nm}$ UV (4 $\mathrm{mW} / \mathrm{cm}^{2}$ ) polymerization was carried out for at least $7 \mathrm{~h}$ to synthesize the $2^{\text {nd }}$ PAAm network within the $1^{\text {st }}$ network gels in an argon blanket. Materials and sample preparation methods for the DN elastomers are described in the Supporting Information.

Mechanical measurements were performed on as-prepared samples unless specifically mentioned. Uniaxial tensile tests were performed on dumbbell-shaped specimens standardized as JIS K6251-7 (12 mm length and $2 \mathrm{~mm}$ width). Data shown in Figure 4 were measured with Tensilon RTC-1310A (Orientec Co.) while data shown in Figures 2 and 3 were measured with Instron 5965 (Instron Co.). Nominal stress, $\sigma$, and deformation ratio, $\lambda$, are defined as force divided by the initial cross-sectional area and sample gauge length divided by the initial length, respectively. Young's modulus is defined as the slope of $\sigma-\lambda$ curves at $1<\lambda<1.1$. Work of extension, $W_{\text {ext }}$, is defined as the area under $\sigma-\lambda$ curves. Tensile velocity was fixed at 100 $\mathrm{mm} / \mathrm{min}$, which corresponds to strain rate of $0.14 \mathrm{~s}^{-1}$. Cyclic tensile tests were performed on the same specimens with the same tensile velocity. As shown in Figure 2(b), the specimen was stretched to the certain $\lambda_{\max }$ and then immediately unloaded. This process was repeated with increasing $\lambda_{\max }$ until sample rupture occurred without any waiting time between the cycles. 


\section{ASSOCIATED CONTENT}

Supporting Information. Materials, elastomer preparation, swelling properties of the PDMAAm network, and mechanical properties of the NPS-DN gels at their swollen state. This material is available free of charge via the Internet at http://pubs.acs.org.

\section{AUTHOR INFORMATION}

\section{Corresponding Author}

* E-mail: tasuku@sci.hokudai.ac.jp

Notes

The authors declare no competing financial interest.

\section{ACKNOWLEDGMENT}

This research was supported by JSPS KAKENHI grant numbers 17H04891 and 17H06144. The Institute for Chemical Reaction Design and Discovery (WPI-ICReDD) was established by World Premier International Research Initiative (WPI), MEXT, Japan.

\section{REFERENCES}

(1) Liu, Y.; Pharr, M.; Salvatore, G. A. Lab-on-Skin: A Review of Flexible and Stretchable Electronics for Wearable Health Monitoring. ACS Nano 2017, 11 (10), 9614-9635. https://doi.org/10.1021/acsnano.7b04898.

(2) Caló, E.; Khutoryanskiy, V. V. Biomedical Applications of Hydrogels: A Review of Patents and Commercial Products. Eur. Polym. J. 2015, 65, 252-267. https://doi.org/10.1016/j.eurpolymj.2014.11.024. (3) Koetting, M. C.; Peters, J. T.; Steichen, S. D.; Peppas, N. A. Stimulus-Responsive Hydrogels: Theory, Modern Advances, and Applications. Mater. Sci. Eng. $R$ Reports 2015, 93, 1-49. https://doi.org/10.1016/j.mser.2015.04.001.

(4) Creton, C. 50th Anniversary Perspective: Networks and Gels: Soft but Dynamic and Tough. Macromolecules 2017, 50 (21), 8297-8316. https://doi.org/10.1021/acs.macromol.7b01698.

(5) Zhao, X. Multi-Scale Multi-Mechanism Design of Tough Hydrogels: Building Dissipation into Stretchy Networks. Soft Matter 2014, 10 (5), 672-687. https://doi.org/10.1039/C3SM52272E.

(6) Gong, J. P.; Katsuyama, Y.; Kurokawa, T.; Osada, Y. Doub6le-Network Hydrogels with Extremely High Mechanical Strength. Adv. Mater. 2003, $15 \quad$ (14), 1155-1158. https://doi.org/10.1002/adma.200304907.

(7) Gong, J. P. Why Are Double Network Hydrogels so Tough? Soft Matter 2010, 6 (12), 2583-2590. https://doi.org/10.1039/b924290b.

(8) Nakajima, T.; Sato, H.; Zhao, Y.; Kawahara, S.; Kurokawa, T.; Sugahara, K.; Gong, J. P. A Universal Molecular Stent Method to Toughen Any Hydrogels Based on Double Network Concept. Adv. Funct. Mater. 2012, 22 (21), 4426-4432. https://doi.org/10.1002/adfm.201200809.

(9) Ahmed, S.; Nakajima, T.; Kurokawa, T.; Anamul Haque, M.; Gong, J. P. Brittle-Ductile Transition of Double Network Hydrogels: Mechanical Balance of Two Networks as the Key Factor. Polymer 2014, 55 (3), 914-923. https://doi.org/10.1016/j.polymer.2013.12.066. (10) Nakajima, T.; Furukawa, H.; Tanaka, Y.; Kurokawa, T.; Osada, Y.; Gong, J. P. True Chemical Structure of Double Network Hydrogels. Macromolecules 2009, 42 (6), 2184-2189. https://doi.org/10.1021/ma802148p.

(11) Nakajima, T. Generalization of the Sacrificial Bond Principle for Gel and Elastomer Toughening. Polym. J. 2017, 49 (6), 477 485. https://doi.org/10.1038/pj.2017.12.

(12) Ducrot, E.; Chen, Y.; Bulters, M.; Sijbesma, R. P.; Creton, C. Toughening Elastomers with Sacrificial Bonds and Watching Them Break. Science 2014, 344 (6180), 186-189. https://doi.org/10.1126/science. 1248494.
(13) Ducrot, E.; Creton, C. Characterizing Large Strain Elasticity of Brittle Elastomeric Networks by Embedding Them in a Soft Extensible Matrix. Adv. Funct. Mater. 2016, 26 (15), 2482-2492. https://doi.org/10.1002/adfm.201504536.

(14) Matsuda, T.; Nakajima, T.; Gong, J. P. Fabrication of Tough and Stretchable Hybrid Double-Network Elastomers Using Ionic Dissociation of Polyelectrolyte in Nonaqueous Media. Chem. Mater. 2019, 31 (10), 3766-3776. https://doi.org/10.1021/acs.chemmater.9b00871.

(15) Murai, J.; Nakajima, T.; Matsuda, T.; Tsunoda, K.; Nonoyama, T.; Kurokawa, T.; Gong, J. P. Tough Double Network Elastomers Reinforced by the Amorphous Cellulose Network. Polymer 2019, 178, 121686. https://doi.org/10.1016/j.polymer.2019.121686.

(16) Na, Y.-H.; Tanaka, Y.; Kawauchi, Y.; Furukawa, H.; Sumiyoshi, T.; Gong, J. P.; Osada, Y. Necking Phenomenon of DoubleNetwork Gels. Macromolecules 2006, 39 (14), 4641-4645. https://doi.org/10.1021/ma060568d.

(17) Webber, R. E.; Creton, C.; Brown, H. R.; Gong, J. P. Large Strain Hysteresis and Mullins Effect of Tough Double-Network Hydrogels. Macromolecules 2007, $40 \quad$ (8), 2919-2927. https://doi.org/10.1021/ma062924y.

(18) Nakajima, T.; Kurokawa, T.; Ahmed, S.; Wu, W.; Gong, J. P. Characterization of Internal Fracture Process of Double Network Hydrogels under Uniaxial Elongation. Soft Matter 2013, 9 (6), 19551966. https://doi.org/10.1039/C2SM27232F.

(19) Yang, W.; Furukawa, H.; Gong, J. P. Highly Extensible Double-Network Gels with Self-Assembling Anisotropic Structure. Adv. Mater. 2008, $20 \quad$ (23), 4499-4503. https://doi.org/10.1002/adma.200801396.

(20) Sun, J.-Y.; Zhao, X.; Illeperuma, W. R. K.; Chaudhuri, O.; Oh, K. H.; Mooney, D. J.; Vlassak, J. J.; Suo, Z. Highly Stretchable and Tough Hydrogels. Nature 2012, 489 (7414), 133-136. https://doi.org/10.1038/nature11409.

(21) Chen, Q.; Zhu, L.; Zhao, C.; Wang, Q.; Zheng J. A Robust, One-Pot Synthesis of Highly Mechanical and Recoverable Double Network Hydrogels Using Thermoreversible Sol-Gel Polysaccharide. Adv. Mater. $\quad$ 2013, $25 \quad$ (30), 4171-4176. https://doi.org/10.1002/adma.201300817.

(22) Bueche, F.; Kinzig, B. J.; Coven, C. J. Polymer Chain Conformations in Bulk Polymers. Polym. Lett. 1965, 3, 399-402. https://doi.org/10.1002/pol.1965.110030511.

(23) Akagi, Y.; Katashima, T.; Sakurai, H.; Chung, U.-I.; Sakai, T. Ultimate Elongation of Polymer Gels with Controlled Network Structure. RSC Adv. 2013, 3 (32), 13251-13258. https://doi.org/10.1039/c3ra41580e.

(24) Itagaki, H.; Kurokawa, T.; Furukawa, H.; Nakajima, T.; Katsumoto, Y.; Gong, J. P. Water-Induced Brittle-Ductile Transition of Double Network Hydrogels. Macromolecules 2010, 43 (22), 94959500. https://doi.org/10.1021/ma101413j.

(25) Argun, A.; Can, V.; Altun, U.; Okay, O. Nonionic Double and Triple Network Hydrogels of High Mechanical Strength. Macromolecules $\quad \mathbf{2 0 1 4}, \quad 47 \quad$ (18), 6430-6440. https://doi.org/10.1021/ma5014176.

(26) Shams Es-haghi, S.; Weiss, R. A. Fabrication of Tough Hydrogels from Chemically Cross-Linked Multiple Neutral Networks. Macromolecules 2016, $49 \quad$ (23), $4980-8987$. https://doi.org/10.1021/acs.macromol.6b02264.

(27) Matsuda, T.; Nakajima, T.; Fukuda, Y.; Hong, W.; Sakai, T.; Kurokawa, T.; Chung, U. Il; Gong, J. P. Yielding Criteria of Double Network Hydrogels. Macromolecules 2016, 49 (5), 1865-1872. https://doi.org/10.1021/acs.macromol.5b02592.

(28) Millereau, P.; Ducrot, E.; Clough, J. M.; Wiseman, M. E.; Brown, H. R.; Sijbesma, R. P.; Creton, C. Mechanics of Elastomeric Molecular Composites. Proc. Natl. Acad. Sci. U. S. A. 2018, 115 (37), 9110-9115. https://doi.org/10.1073/pnas.1807750115.

(29) James, H. M.; Guth, E. Theory of the Elastic Properties of Rubber. J. Chem. Phys. 1943, 11 (10), 455-481. https://doi.org/10.1063/1.1723785.

(30) Ueda, A.; Nagai, S; In Polymer Handbook, 4th ed.; Brandrup, J., Immergut, E. H., Grulke, E. A., Menges, G., Eds.; Wiley-Interscience: New York, 1999; p II-110- II-145. 
(31) Kwon, H. J.; Yasuda, K.; Ohmiya, Y.; Honma, K.; Chen, Y. M.; Gong, J. P. In Vitro Differentiation of Chondrogenic ATDC5 Cells Is Enhanced by Culturing on Synthetic Hydrogels with Various Charge
Densities. Acta Biomater. 2010, 6

(2), 494-501. 